\title{
Transcriptional repression of ANGPT1 by histone H3K9 demethylase KDM3B
}

\author{
Arim Han , Yun-Cheol Chae , Jin Woo Park, Kee-Beom Kim, Ji-Young Kim E Sang-Beom Seo * \\ Department of Life Science, College of Natural Sciences, Chung-Ang University, Seoul 156-756, Korea
}

\begin{abstract}
Here we report that the $\mathrm{H} 3 \mathrm{~K} 9$ demethylase KDM3B represses transcription of the angiogenesis regulatory gene, ANGPT1. Negative regulation of $A N G P T 1$ by KDM3B is independent of its Jumonji (JmjC) domain-mediated H3K9 demethylase activity. We demonstrate that KDM3B downregulates ANGPT1 via interaction with SMRT, and suggest that the repressor complex is formed at the promoter area of ANGPT1. Using MTT and wound healing assays, depletion of KDM3B was found to increase cell proliferation and cell motility, indicating that KDM3B has a role in angiogenesis. [BMB Reports 2015; 48(7): 401-406]
\end{abstract}

\section{INTRODUCTION}

Chromatin is a highly common structure that contains DNA, histones, and other chromosomal proteins. The basic building block of chromatin is the nucleosome, which is made up of two copies of each histone: $\mathrm{H} 2 \mathrm{~A}, \mathrm{H} 2 \mathrm{~B}, \mathrm{H} 3$, and $\mathrm{H} 4$. The $\mathrm{N}$-terminal tails of the histones are subject to posttranslational modifications (PTMs), which in turn affect numerous biological processes including transcription, replication, and chromosome maintenance. Methylation of the lysine residues within histones is regulated by methyltransferases (KMTs) and demethylases (KDMs) for the maintenance of cell fate and genomic stability. KDM3B, also known as JMJD1B, is a histone H3K9-specific demethylase. Its gene is located in the $5 q 31$ chromosomal region in humans, which has often been found to be deleted in breast cancer (1) and myeloid leukemia (2). KDM3B has been studied for regulation of the expression of the leukemic oncogene Imo2 (3).

Angiogenesis, the process by which blood capillaries grow from the pre-existing vascular tree, is a key event in tumor

*Corresponding author. Tel: +82-2-820-5242; Fax: +82-2-822-3059; E-mail: sangbs@cau.ac.kr

${ }^{\text {"}}$ These authors contributed equally to this work.

http://dx.doi.org/10.5483/BMBRep.2015.48.7.188

Received 1 September 2014, Revised 28 September 2014, Accepted 19 November 2014

Keywords: ANGPT1, Cell motility, KDM3B, SMRT, Transcription growth and progression. Among the different growth factors which play roles in angiogenesis, VEGFA (vascular endothelial growth factor A) and its receptor are major mediators of tumor angiogenesis. Angiopoietin-1 (ANGPT1) is fundamental to physiological angiogenesis, including endothelial cell survival, vascular branching, and pericyte recruitment (4). ANGPT1 binds to and activates the TEK/TIE2 receptor by inducing its dimerization and tyrosine phosphorylation, playing an important role in the regulation of angiogenesis $(5,6)$. Overexpression of ANGPT1 has been proven to occur in malignant glioblastoma $(7,8)$, neuroblastoma (9), non-small cell lung cancer (10), and other tumors (4).

However, the role of ANGPT1 in tumor angiogenesis is less clear. Increasing amounts of experimental data have suggested that ANGPT1 contributes to the stabilization of newly organized blood vessels (11).

In the present study, we report that KDM3B negatively regulates ANGPT1 transcription with its corepressor, SMRT, via recruitment to the ANGPT1 promoter. This activity is independent of its demethylase activity, suggesting that KDM3B has a mechanism by which to repress transcription of the target gene ANGPT1 to inhibit cellular proliferation and migration.

\section{RESULTS}

Negative regulation of $A N G P T 1$ transcription by KDM3B Previously, we conducted global gene expression profiling of human erythroleukemic K562 cells stably expressing KDM3B shRNA in the Tet-on inducible expression system (3). Among the differentially expressed genes, ANGPT1 was found to be up-regulated when KDM3B was knocked down in the K562 cell line (3). As a key regulator of angiogenesis, ANGPT1 binds to and activates the TEK/TIE2 receptor by inducing tyrosine phosphorylation. Since the transcriptional activation activity of KDM3B has been known to be conferred through $\mathrm{H} 3 \mathrm{~K} 9$ demethylation, we wanted to investigate the mechanism of the KDM3B-mediated negative regulation of ANGPT1. First, RT-PCR was performed on the K562 shKDM3B stable cell line to confirm the microarray data. As expected, the transcription of ANGPT1 was up-regulated by KDM3B knock-down (Fig. 1A). To further confirm the ANGPT1 up-regulation induced by the depletion of KDM3B, RT-PCR was also conducted on 293T cells. Again, ANGPT1 was increased in 293T cells when

ISSN: 1976-670X (electronic edition)

Copyright (C) 2015 by the The Korean Society for Biochemistry and Molecular Biology

(c) This is an open-access article distributed under the terms of the Creative Commons Attribution Non-Commercial License (http://creativecommons.org/licenses/by-nc/3.0) which permits unrestricted non-commercial use, distribution, and reproduction in any medium, provided the original work is properly cited. 
A
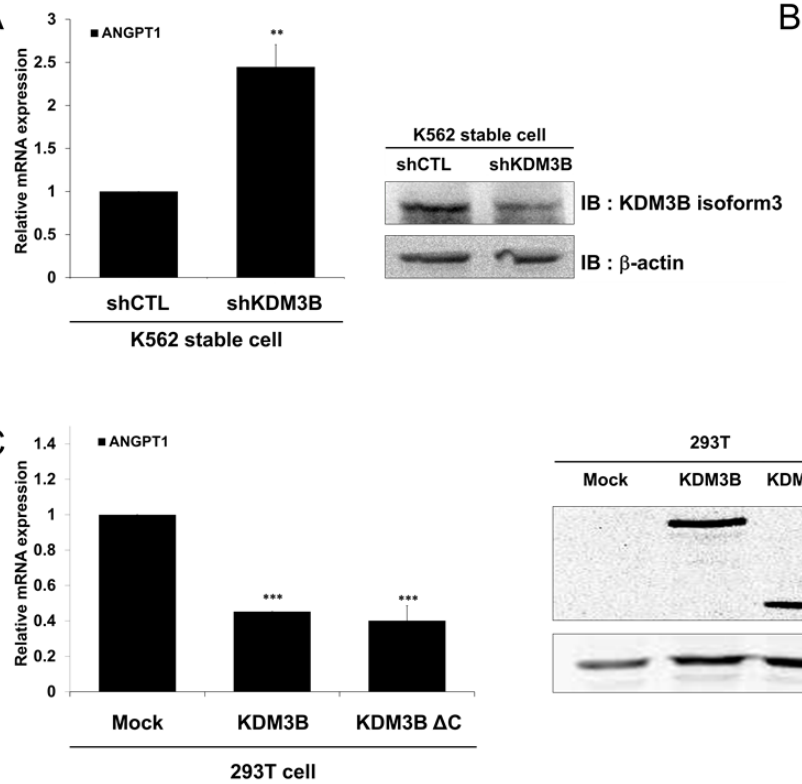
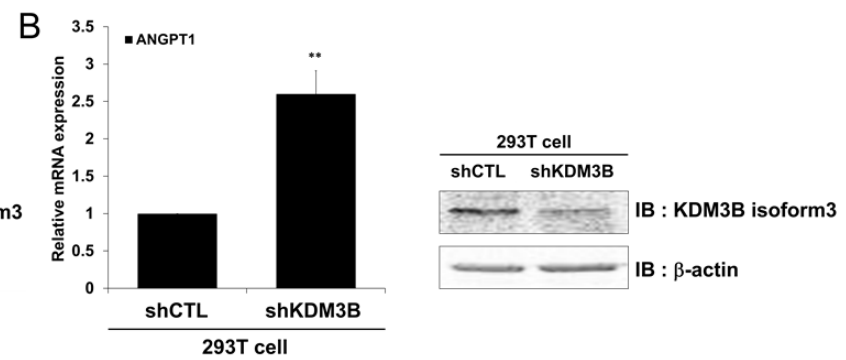

Fig. 1. KDM3B represses ANGPT1 expression. (A) K562 shKDM3B stable cells were analyzed by real time PCR to examine the mRNA expression level of ANGPT1. Results are shown as means \pm SDs; $n=3$. ${ }^{* *} \mathrm{P}<0.01$. KDM3B knock-down was confirmed by western blot analysis. (B) shKDM3B transfected 293T cells were analyzed by real time PCR to examine the mRNA expression levels of ANGPT1. Results are shown as means \pm SDs; $\mathrm{n}=3 . * * \mathrm{P}<0.01$. KDM3B knock-down was confirmed by western blot analysis. (C) GFP-KDM3B or GFP-KDM3B $\triangle \mathrm{C}$ transfected 293T cells were analyzed by real time PCR to examine the mRNA expression levels of $A N G P T 1$. Overexpression of $\mathrm{KDM} 3 \mathrm{~B}$ and $\mathrm{KDM} 3 \mathrm{~B} \Delta \mathrm{C}$ confirmed by western blot analysis. Results are shown as means $\pm \mathrm{SDs} ; \mathrm{n}=3$. $* * * \mathrm{P}<$ 0.001 .

KDM3B was knocked down by transient transfection with KDM3B shRNA (Fig. 1B).

To investigate whether KDM3B regulates ANGPT1 through its demethylase activity, transfection was carried out with $\mathrm{KDM} 3 \mathrm{~B}$ or $\mathrm{KDM} 3 \mathrm{~B} \Delta \mathrm{C}$, in which demethylase activity of jmjC-domain was deleted. When KDM3B was overexpressed, significant decrease of ANGPT1 was observed. Notably, ANGPT1 was also decreased in cells overexpressing $\mathrm{KDM} 3 \mathrm{~B} \Delta \mathrm{C}$ (Fig. 1C). These results indicated that the KDM3B-mediated down-regulation of ANGPT1 was independent of its demethylase activity.

\section{Transcriptional regulation of $A$ NGPT1 by KDM3B}

In order to determine the mechanism of ANGPT1 down-regulation by KDM3B more precisely, a reporter assay was performed using the ANGPT1 promoter-driven luciferase (luc) reporter system. Consistent with the results of microarray analysis, the transcriptional activity of ANGPT1 was increased in a dose-dependent manner with the knockdown of KDM3B in 293T cells (Fig. 2A). In addition, ANGPT1 was decreased when KDM3B was overexpressed (Fig. 2B). As mentioned above, KDM3B activates gene expression through the demethylation of $\mathrm{H} 3 \mathrm{~K} 9 \mathrm{me} 1 / 2$. However, the regulation of ANGPT1 indicated the opposite results. Thus, we hypothesized that oth- er transcription factors were involved in the regulation of ANGPT1.

Having established that KDM3B negatively regulates the transcription of ANGPT1, we next searched for any transcription factor which is involved in the KDM3B-mediated transcriptional regulation. Transcription factors known for their roles in angiogenesis were screened, such as JunB, and GATA1. Each transcription factor was then tested in ANGPT1 transcriptional activity reporter analysis.

As shown in Fig. 2C, KDM3B knockdown increased ANGPT1 transcription, which was further increased by the addition of JunB (Fig. 2C). Similar results were obtained when another transcription factor, GATA1, was added (Fig. 2D). These results indicated that JunB and GATA1 are involved in the transcriptional activation of ANGPT1. However, further activation of the ANGPT1 promoter activity despite KDM3B-mediated transcriptional repression suggests that JunB and GATA1 regulate ANGPT1 via a different pathway from KDM3B.

\section{KDM3B interacts with corepressor SMRT}

Since our results indicated that KDM3B functions as a transcriptional repressor for $A N G P T 1$, we decided to investigate whether KDM3B works in concert with corepressors such as HDAC, NcoR, or SMRT to repress ANGPT1. To accomplish 
A

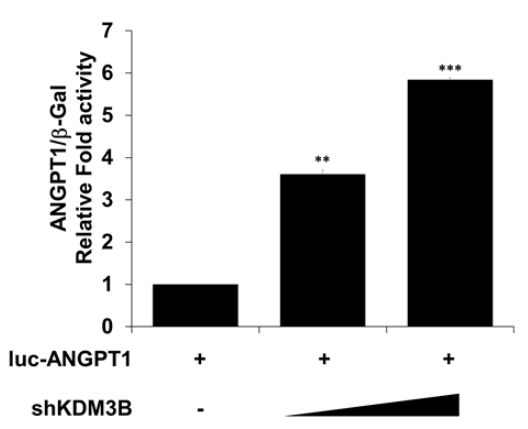

C

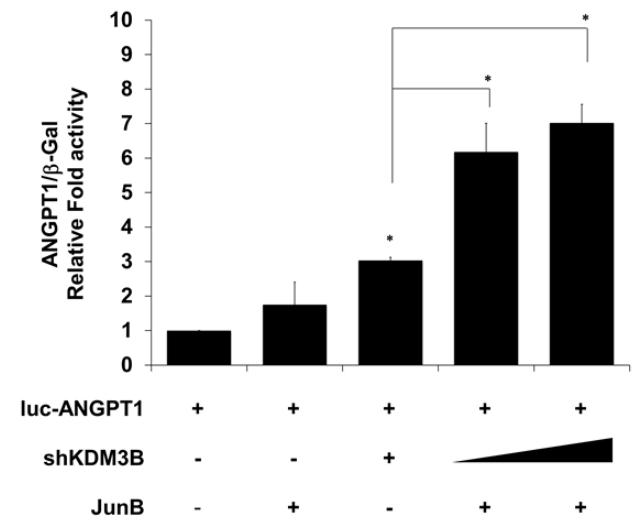

B
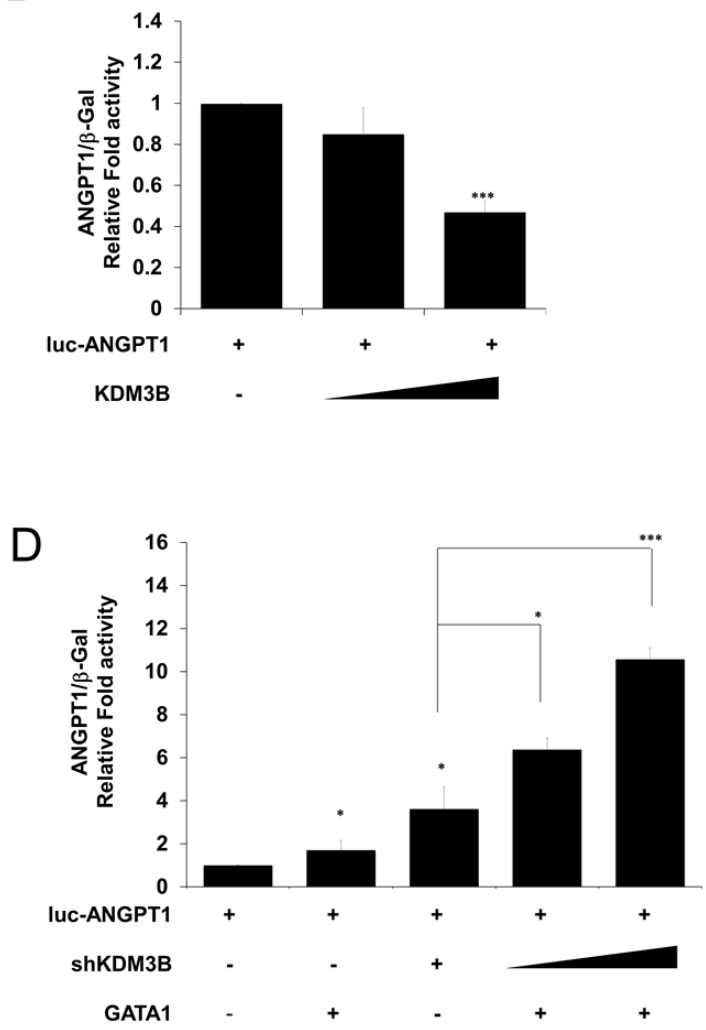

Fig. 2. KDM3B regulates ANGPT1 transcription. (A and B) shKDM3B (A) or KDM3B (B) were co-transfected with the ANGPT1 promoter-luc construct in 293T cell line. Cell extracts were then assayed for luciferase activity, which was normalized to $\beta$-galactosidase. Results are shown as means \pm SDs; $\mathrm{n}=3$. ${ }^{* * * P}<0.001 ; * * \mathrm{P}<0.01$. (C and D) 293T cells were co-transfected with the indicated plasmids. Cell extracts were then assayed for luciferase activity, which was normalized to $\beta$-galactosidase. Results are shown as means \pm SDs; $\mathrm{n}=3$. $* * * \mathrm{P}<0.001 ; * \mathrm{P}<0.05$.

this, transient transfection and Immunoprecipitations assays were conducted. These assays clearly showed that SMRT, but not HDAC or NcoR1 (data not shown), strongly interacted with KDM3B, suggesting that KDM3B and SMRT form a repressor complex during ANGPT1 transcription (Fig. 3A). To further test the SMRT-mediated KDM3B activity, reporter assays were carried out to investigate whether KDM3B works together with SMRT. As expected, overexpression of both wild type KDM3B and that with the jmjC-domain deleted repressed ANGPT1 transcription (Fig. 3B). When cells were cotransfected with KDM3B and SMRT, ANGPT1 transcription was further downregulated (Fig. 3B). However, when cells were cotransfected with KDM3B and shSMRT, downregulation of ANGPT1 was recovered (Fig. 3B). Next, we knocked down KDM3B with shRNA and ANGPT1 transcription was upregulated (Fig. 3C). Furthermore, when cells were cotransfected with shKDM3B and shSMRT, ANGPT1 transcription was further upregulated (Fig. 3C). These results suggest that KDM3B and SMRT form a corepressor complex and negatively regulate ANGPT1 transcription.

\section{Increase of cell motility by depletion of KDM3B}

Previous studies reported induction of ANGPT1 increase cell proliferation, wound healing, and invasion (6, 12-14). To further investigate the physiological effects of the KDM3B- mediated transcriptional regulation of ANGPT1, we first conducted the cell proliferation assay (Fig. 4A). When KDM3B was overexpressed, a decrease in cell proliferation was observed. However, cellular proliferation was significantly increased when KDM3B was knocked down.

Previous studies suggested that ANGPT1 contributes to angiogenesis by stabilizing blood vessel maturation (11). A scratch-motility assay was then performed using 293T cells transiently expressing KDM3B or shKDM3B with either control pCMV-3X-flag or shCTL. The closure of wounded areas was observed to be significantly accelerated in KDM3B knock-down 
A

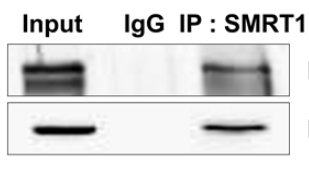

IB : SMRT

IB : Flag (KDM3B)

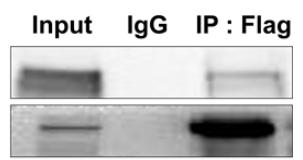

IB : SMRT

IB : Flag (KDM3B)

C

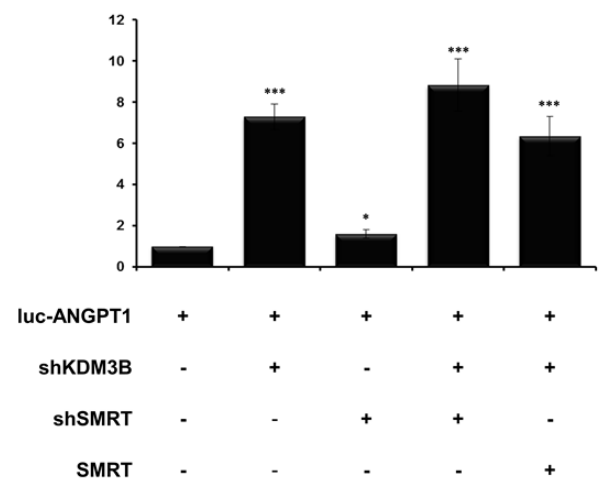

cells suggesting that KDM3B plays a role in the repression of cell motility, probably via regulation of ANGPT1 (Fig. 4B). As expected, the closure of wounds was decelerated when KDM3B was overexpressed. These results indicated that increase of ANGPT1 induced by knock-down of KDM3B may have regulated cell motility in our assay. Collectively, our findings indicate that angiogenesis-mediated wound healing is regulated through the involvement of a subset of KDM3B target genes exemplified by ANGPT1, and thereby affects cell proliferation and cell motility properties.

\section{DISCUSSION}

KDM3B is a H3K9me1/2 demethylase, and is highly expressed in hematopoietic cells (15). H3K9 methylation status is linked to heterochromatin formation and the silencing of target genes $(16,17)$. Herein, we attempted to elucidate the negative regulation of ANGPT1 transcription by KDM3B. Consistent with our previous microarray analysis, depletion of KDM3B increased the expression of ANGPT1. By reporter assay using the
B
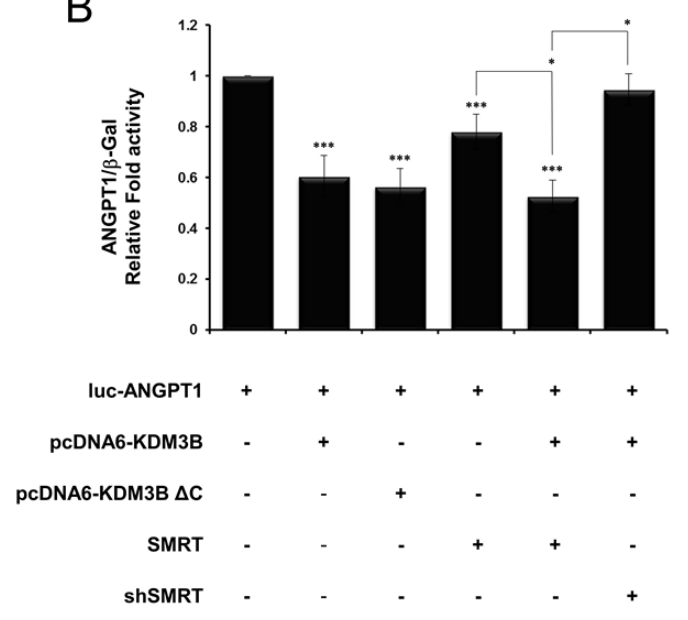

Fig. 3. KDM3B interacts with corepressor SMRT. (A) 293T cells were co-transfected with Flag-KDM3B and pCMX-SMRT prior to immunoprecipitation with anti-Flag antibodies. Immunoprecipitated and associated proteins were eluted, resolved by SDS-PAGE, and immunoblotted with anti-Flag and anti-SMRT antibodies. (B and C) 293T cells were co-transfected with the indicated plasmids. Cell extracts were then assayed for luciferase activity, which was normalized to $\beta$-galactosidase. Results are shown as means \pm SDs; $\mathrm{n}=3$. ${ }^{* * *} \mathrm{P}<0.001 ; * \mathrm{P}<0.05$.

ANGPT1 promoter, it was found that ANGPT1 promoter activity was up-regulated when KDM3B was knocked down. The negative regulation of $A N G P T 1$ by KDM3B was demonstrated to be independent of the H3K9 demethylase activity of KDM3B. There have been reports that G9a positively regulates the transcription mediated by various nuclear receptors through an HMTase-independent mechanism $(18,19)$. Previously, we reported that H3K9 HMTase G9a activates the transcription of p21 in p53-null H1299 cells independent of its HMTase activity (20). These reports, including the current study, indicate that there are different layers of regulatory networks in epigenetic modifiers, including HMTases and histone demethylases. It is also interesting that up-regulation of the ANGPT1 transcription level was independent of angiogenesis-related transcription factors such as JunB and GATA1, tested herein. Further studies will be needed to elucidate the fine mechanism of transcriptional regulation by $\mathrm{KDM} 3 \mathrm{~B}$, including identification of the responsible transcription factors involved.

Furthermore, we found that wound healing and invasion were increased by the knock-down of KDM3B. These results 

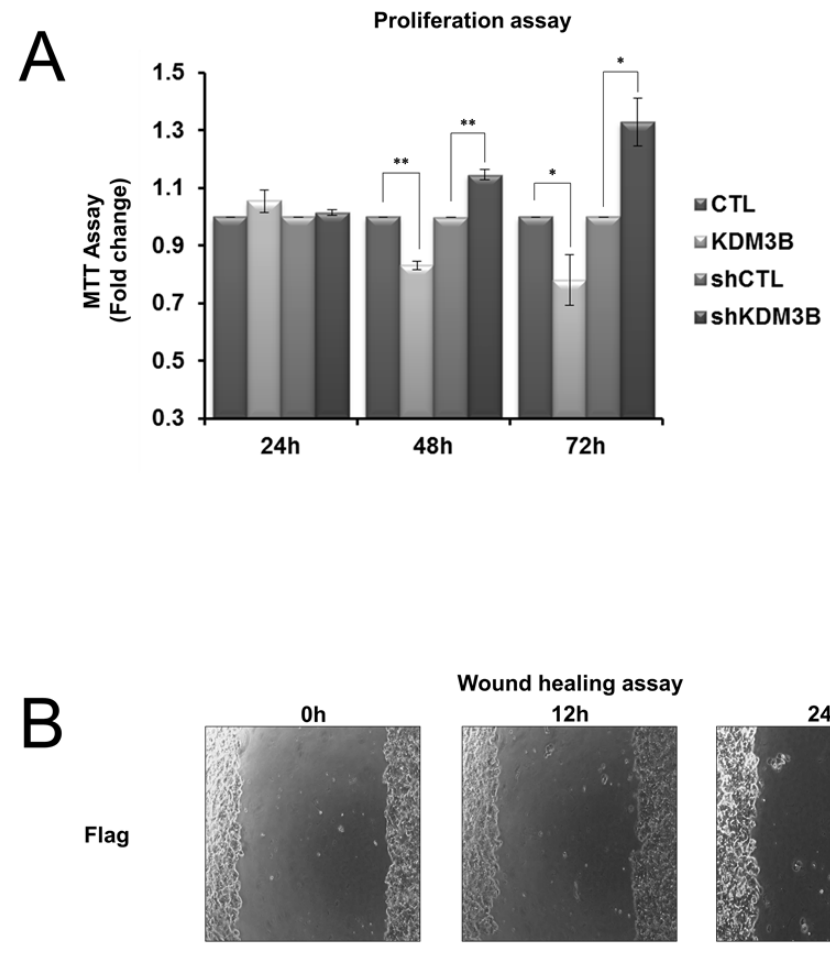

Wound healing assay
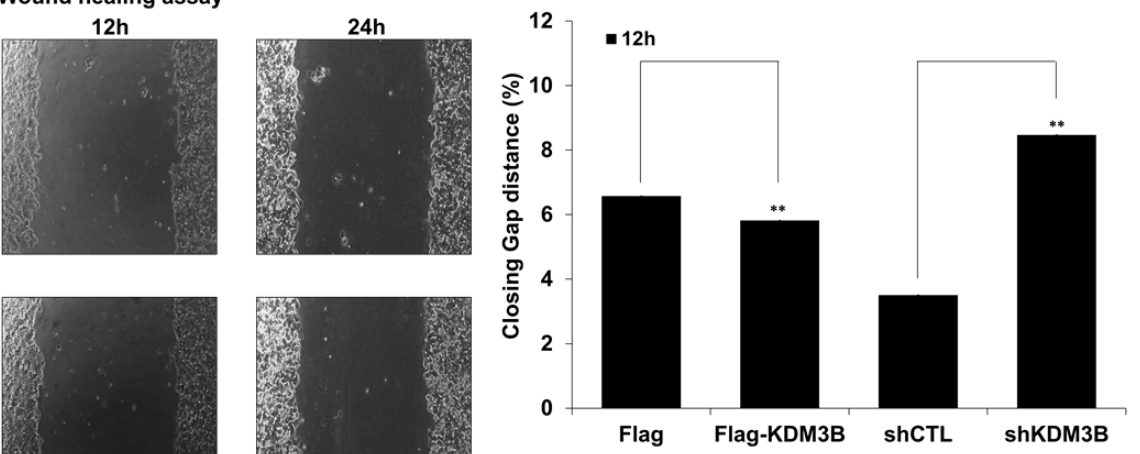

Flag-KDM3B
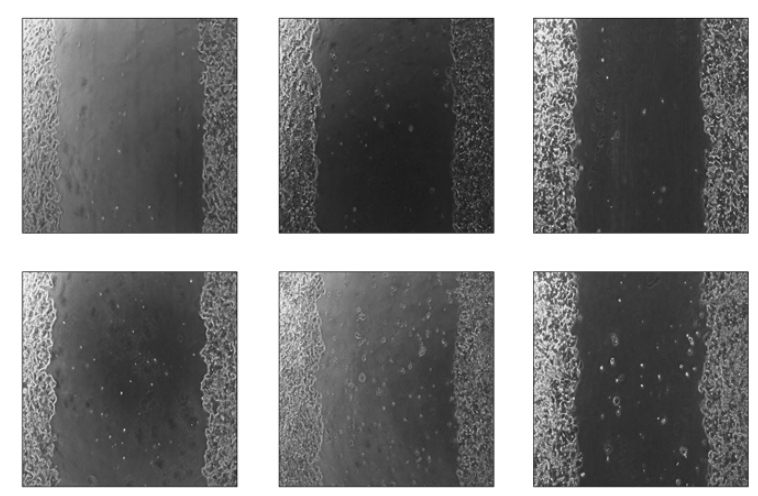

Fig. 4. Depletion of KDM3B induces cell proliferation and motility. (A) Proliferation was monitored over $72 \mathrm{~h}$ in $293 \mathrm{~T}$ cells expressing FlagKDM3B or shKDM3B. (B) Photomicrographs from a scratch-motility assay of MCF7 expressing FlagKDM3B or shKDM3B for $24 \mathrm{~h}$ (Left panel). Percent of the gap distance closed between the two migrating form fronts was measured and shown as a graph (Right panel). Results are shown as means \pm SDs; $\mathrm{n}=3$. ${ }^{*} \mathrm{P}<0.05 ; * * \mathrm{P}<0.01$.

indirectly suggest that KDM3B plays a role in cell migration and proliferation. In conclusion, depletion of KDM3B increased the transcription of ANGPT1. Down-regulation of ANGPT1 was independent of the demethylase activity of $\mathrm{KDM} 3 \mathrm{~B}$, and the SMRT corepressor plays role in the transcriptional repression of $A N G P T 1$ by KDM3B.

\section{MATERIALS AND METHODS}

Cell culture and transient transfection, RNA isolation and real-time PCR, Western blot analysis, Immunoprecipitation, and Statistical analysis are described in the online data supplement, available at http://www.bmbreports.org/. 


\section{Luciferase assay}

293T and HCT116 cells were seeded at the density of $6 \times 10^{4}$ cells in 48-well plates and transfected with pGL3-ANGPT1 promoter (100 ng), shKDM3B (200 ng or $400 \mathrm{ng}$ ), shSMRT (300 ng), GFP-KDM3B (100 ng or 200 ng), pCMV10-Flag-Imo2 (100 ng), and pCMX-PL1-JunB (50 ng) using PEI. After $48 \mathrm{~h}$ of transfection, the cells were collected and lysed in cell culture lysis reagent. Luciferase activities were then determined by adding $10 \mu \mathrm{l}$ of luciferase assay substrate into $70 \mu \mathrm{l}$ of cell lysates, then measuring using a Glomax luminometer (Promega). The results were confirmed by performing each experiment at least in triplicate.

\section{MTT(3-(4,5-dimethylthiazol-2-yl)-2,5-diphenyltetrazolium bromide) assay}

293T cells were seeded in 48-well plates $\left(8 \times 10^{4}\right.$ cells/well) and transiently transfected with pCMV10-Flag-KDM3B and shKDM3B. After $0-4$ days of incubation at $37^{\circ} \mathrm{C}, 20 \mu \mathrm{l}$ of MTT $(1 \mathrm{mg} / \mathrm{ml})$ was added to the each well and incubated for an additional $4 \mathrm{~h}$ at $37^{\circ} \mathrm{C}$, followed by aspiration of the medium and addition of $200 \mu \mathrm{l}$ of dimethyl sulfoxide (DMSO). OD values were determined with an ELISA reader (Biochrom) at the wavelength of $570 \mathrm{~nm}$. DMSO alone was measured as the blank and subtracted from all values to correct for background in the measurements.

\section{Wound healing assay}

Cells were seeded in 24-well plates and transfected with pLKO. 1 and shKDM3B. Twenty four hours after transfection, the cells were located in monolayers, and were scratched once with medium using a pipette tip to obtain a "scratch" in each well. Cells were then washed once with medium. Migration was monitored every $12 \mathrm{~h}$, and photomicrographs were taken at each time point.

\section{ACKNOWLEDGEMENTS}

This study was supported by the National R\&D Program for Cancer Control, Ministry of Health \& Welfare (1020150).

\section{REFERENCES}

1. Mar BG, Bullinger L, Basu E et al (2012) Sequencing histone-modifying enzymes identifies UTX mutations in acute lymphoblastic leukemia. Leukemia 26, 1881-1883

2. MacKinnon RN, Kannourakis G, Wall M and Campbell LJ (2011) A cryptic deletion in 5q31.2 provides further evidence for a minimally deleted region in myelodysplastic syndromes. Cancer Genet 204, 187-194

3. Kim JY, Kim KB, Eom GH et al (2012) KDM3B is the H3K9 demethylase involved in transcriptional activation of Imo2 in leukemia. Mol Cell Biol 32, 2917-2933

4. Metheny-Barlow LJ and Li LY (2003) The enigmatic role of angiopoietin-1 in tumor angiogenesis. Cell Res 13, 309-317

5. Sato A, Iwama A, Takakura N, Nishio H, Yancopoulos
GD and Suda T (1998) Characterization of TEK receptor tyrosine kinase and its ligands, Angiopoietins, in human hematopoietic progenitor cells. Int Immunol 10, 1217-1227

6. Davis S, Aldrich TH, Jones PF et al (1996) Isolation of angiopoietin-1, a ligand for the TIE2 receptor, by secretion-trap expression cloning. Cell 87, 1161-1169

7. Stratmann A, Risau W and Plate KH (1998) Cell type-specific expression of angiopoietin-1 and angiopoietin-2 suggests a role in glioblastoma angiogenesis. Am J Pathol 153, 1459-1466

8. Ding H, Roncari L, Wu X et al (2001) Expression and hypoxic regulation of angiopoietins in human astrocytomas. Neuro Oncol 3, 1-10

9. Eggert A, Ikegaki N, Kwiatkowski J, Zhao H, Brodeur GM and Himelstein BP (2000) High-level expression of angiogenic factors is associated with advanced tumor stage in human neuroblastomas. Clin Cancer Res 6, 1900-1908

10. Hatanaka H, Abe Y, Naruke M et al (2001) Significant correlation between interleukin 10 expression and vascularization through angiopoietin/TIE2 networks in non-small cell lung cancer. Clin Cancer Res 7, 1287-1292

11. Papapetropoulos A, Garcia-Cardena G, Dengler TJ et al (1999) Direct actions of angiopoietin-1 on human endothelium: evidence for network stabilization, cell survival, and interaction with other angiogenic growth factors. Lab Invest 79, 213-223

12. Parborell F, Abramovich D, Irusta G and Tesone M (2011) Angiopoietin 1 reduces rat follicular atresia mediated by apoptosis through the PI3K/Akt pathway. Mol Cell Endocrinol 343, 79-87

13. Holopainen T, Huang $H$, Chen $C$ et al (2009) Angiopoietin-1 overexpression modulates vascular endothelium to facilitate tumor cell dissemination and metastasis establishment. Cancer Res 69, 4656-4664

14. Cho CH, Sung HK, Kim KT et al (2006) COMP-angiopoietin-1 promotes wound healing through enhanced angiogenesis, lymphangiogenesis, and blood flow in a diabetic mouse model. Proc Natl Acad Sci U S A 103, 4946-4951

15. Yoshimi A and Kurokawa M (2011) Key roles of histone methyltransferase and demethylase in leukemogenesis. J Cell Biochem 112, 415-424

16. Feldman N, Gerson A, Fang J et al (2006) G9a-mediated irreversible epigenetic inactivation of Oct-3/4 during early embryogenesis. Nat Cell Biol 8, 188-194

17. Lachner M, O'Carroll D, Rea S, Mechtler $K$ and Jenuwein T (2001) Methylation of histone H3 lysine 9 creates a binding site for HP1 proteins. Nature 410, 116-120

18. Purcell DJ, Jeong KW, Bittencourt D, Gerke DS and Stallcup MR (2011) A distinct mechanism for coactivator versus corepressor function by histone methyltransferase G9a in transcriptional regulation. J Biol Chem 286, 41963 $-41971$

19. Lee DY, Northrop JP, Kuo MH and Stallcup MR (2006) Histone H3 lysine 9 methyltransferase G9a is a transcriptional coactivator for nuclear receptors. J Biol Chem 281, 8476-8485

20. Oh ST, Kim KB, Chae YC, Kang JY, Hahn Y and Seo SB (2014) H3K9 histone methyltransferase G9a-mediated transcriptional activation of p21. FEBS Lett 588, 685-691 